\title{
Mass and Stiffness Impact on the Middle Ear and the Cochlear Partition
}

\author{
Jinsook Kim and Miseung Koo \\ Division of Speech Pathology and Audiology, Research Institute of Audiology and Speech Pathology, College of Natural Sciences, \\ Hallym University, Chuncheon, Korea
}

Received March 25, 2015

Revised March 30, 2015

Accepted March 31, 2015

\author{
Address for correspondence \\ Jinsook Kim, FAAA, PhD \\ Division of Speech Pathology and \\ Audiology, Research Institute of \\ Audiology and Speech Pathology, \\ College of Natural Sciences, \\ Hallym University, \\ 1 Hallymdaehak-gil, \\ Chuncheon 200-702, Korea \\ Tel +82-33-248-2213 \\ Fax $+82-33-256-3420$ \\ E-mail jskim@hallym.ac.kr
}

\begin{abstract}
Mass and stiffness affect on the peculiar characteristics of transmission of the middle ear and the distinctive behavior of the cochlear mechanics. Applying the principle of the mass and stiffness, the band-pass characteristic transfer function of the middle ear has been explained. The greatest transfer function of the middle ear, approximately $24-29 \mathrm{~dB}$, is observed at $1-2 \mathrm{kHz}$ in both cat and human species. However, at lower frequencies, the transfer function was disturbed by the stiffness of the middle ear primarily due to middle ear cavity. At higher frequencies, the transfer function was disturbed by the stiffness of the middle ear primarily due to middle ear bones. Several examples, such as an acoustic reflex, otitis media, and otosclerosis are discussed. For understanding the traveling wave of the basilar membrane, different place tuning at certain stimulus frequencies, contrastingly shaped basilar membrane to the cochlear duct, and the structural and physical characteristics of the whole cochlear partition were reviewed in terms of changing width, mass, and stiffness from the base to apex. Being about ten times wider, more massive, and one hundredfold stiffer at the base than the apex, the nature of the cochlear partition to absorb high-frequency energy changes in fluid pressure declines toward the apex. Consequently, at the base of the cochlea, high frequencies stimuli are decoded while low frequencies stimuli are decoded at the apex of the cochlea. Due to these characteristics of the cochlear partition, the direction of the traveling wave was also proved to be in the fashion of base-to-apex always.
\end{abstract}

J Audiol Otol 2015;19(1):1-6

KEY WORDS: Mass · Stiffness · Middle ear · Transfer function · Cochlear partition.

\section{Introduction}

There are two auditory systems in human hearing mechanism, the peripheral and the central systems. The peripheral auditory system can be divided into three parts and the simplified physiology is as followed. First, the outer ear adjusts the sound wave in transferring the acoustic vibrations to the eardrum with changing of the sound pressure depending on the direction of the sound and resonating the sound pressure in the range of frequencies of $2-7 \mathrm{kHz}$. Secondly, the middle ear couples the sound energy from the low-impedance air to the

This is an Open Access article distributed under the terms of the Creative Commons Attribution Non-Commercial License (http://creativecommons. org/licenses/by-nc/3.0/) which permits unrestricted non-commercial use, distribution, and reproduction in any medium, provided the original work is properly cited. higher impedance cochlear fluids acting as a transformer. Thirdly, the inner ear, also known as the cochlea, separates stimulus frequencies into different spatial regions and converts changes in pressure into vibration for the discharge of auditory neurons. Directly responsible for sensory function in the cochlea, the organ of Corti sits on the top of the basilar membrane. A cellular and gelatinous structure, tectorial membrane, covers top of the organ of Corti. These three essential structures for cochlear function, the basilar membrane, the organ of Corti, and the tectorial membrane make up the cochlear partition. Mass and stiffness with the other physical properties of the middle ear and the cochlear partition affect on the peculiar characteristics of transmission of the middle ear and the behavior to different frequencies of stimulation in the cochlear partition.

The effectiveness of transmitting vibration of the sound en- 
ergy is frequency dependent because every object has a resonant frequency. The resonant frequency is determined by an object's acoustic impedance limiting a certain frequency transmission. We manipulate this acoustic impedance when we play a musical instrument. Two renowned properties that determine an object's resonant frequency are mass and stiffness. Mass limits high-frequency transmission, as heavier objects vibrate at lower frequencies while stiffness limits low-frequency transmission, as a less elastic in other words stiff objects vibrate at higher frequencies. For example, a xylophone uses bigger keys for resonance of lower frequencies and in flute, the shorter the column, the resonant frequency becomes higher as changing the length of an air column changes its elasticity. In the peripheral auditory system, the middle ear and the cochlear partition are affected by the mass and stiffness for their unique movement characteristics. When the sound energy transmits in the middle ear, the bones supply mass and volume of the middle ear cavity impinges on stiffness providing bandpass characteristics for the transfer function. Also, depending on its structural nature, the degree of the establishment of gradients for mass and stiffness of the cochlear partition is responsible for the manner of the traveling wave and the distinctive basilar movement.

\section{Mass and Stiffness of the Middle Ear}

\section{Transfer function of the middle ear}

The middle ear transmits acoustic energy from the external auditory meatus to the cochlea. When the sound enters the middle ear cavity, its acoustic energy is converted into the mechanical energy, then into hydraulic energy when entered to cochlear fluids. In response to much higher impedance of the cochlear fluids, the middle ear allows impedance matching by increasing the pressure. This is called the transfer function of the middle ear. This phenomenon is mainly attributed to two principles. One is the ratio of the area of the tympanic membrane and the oval window and the other is the lever action of two middle ear bones, the malleus and the incus. As the middle ear transforms the sound pressure variation of the ear canal into a sound pressure variation in the scala vestibuli, the transfer function can be shown by plotting the ratio of the two pressures at different stimulus frequencies.

The transfer function was measured by the pressure change from the tympanic membrane to the cochlear duct of the cat, just behind the oval window [1,2]. Performing corresponding measurements in human cadaver temporal bones, the pressure gain curve of the middle ear through the transfer function was also obtained [3,4]. In the two species, the sound pressures are approximately 24 and $29 \mathrm{~dB}$ greater at the cochlear duct than

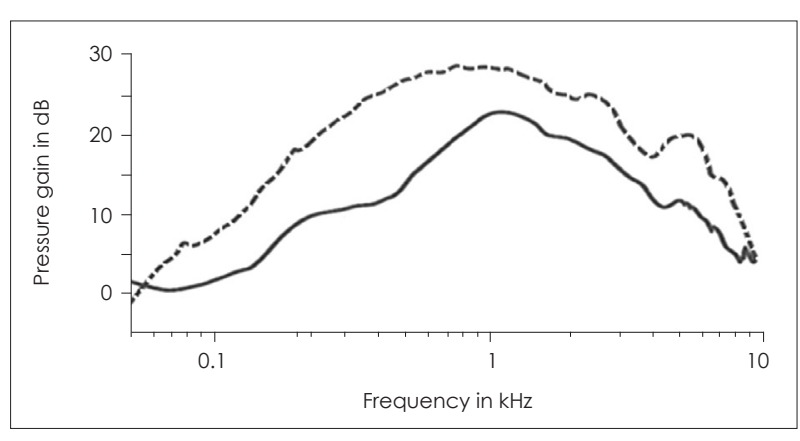

Fig. 1. The transfer function of the middle ear for both cat (dotted line) and human (solid line) as a function frequency.

those at the tympanic membrane. The pressure gains were different across band-pass. The greatest pressure gain for both cats and human beings appeared around $1-2 \mathrm{kHz}$ and smoothly decline toward low and high frequencies. Slightly increased pressure at $4 \mathrm{kHz}$ had been proved to be relevant to acoustic resonances in the bulla of the middle ear cavity (Fig. 1). Then, why does the transfer function of the middle ear show band-pass characteristic. If not completely, mass and stiffness properties of the middle ear structure seem to explain this phenomenon.

\section{Mass and stiffness impact on the transmission loss}

Forced vibrations and a resonant frequency of a vibrating object are determined by mass and stiffness. Mass helps lowfrequency vibration and disturbs transmitting high-frequency sound while stiffness helps high-frequency vibration and disturbs transmitting low-frequency sound. Applying this principle, we can attempt to identify some of the factors governing the band-pass characteristics of the transfer function of the middle ear. The reduced transmission of low-frequency sound is affected by an elastic stiffness which has been ascribed to elasticity in the tympanic membrane, the ligaments of the middle ear bones, and a compression and expansion of the air in the middle ear cavity. When the tympanic membrane moves in and out, air in the middle ear cavity is compressed and expanded reducing the movement of the tympanic membrane. The elastic stiffness is particularly important at low frequencies because of the mathematical relation between the pressure of the sound wave and the displacement of the air. The degree of displacement produced by a compression and expansion of the air in the middle ear cavity is the reciprocal of frequency, as seen in the equation presented below. The following equation is an expression that calculates the value of displacement. Where ' $\mathrm{d}$ ' is the displacement, ' $\mathrm{f}$ ' is the frequency, ' $\mathrm{I}$ ' is the intensity, and ' $\mathrm{z}$ ' is the impedance. As the frequency drops, a comparatively large displacement will be produced deriving elastic stiffness in the middle ear structure [5]. 


$$
d=\left(\frac{1}{2 \pi f}\right) \sqrt{\left(\frac{2 I}{z}\right)}
$$

Although the reduction in transmission of high-frequency sound is affected by many factors and their relative factors are not known, the mass of the middle ear bones is thought to be a significant impact on disturbing transmissions of high frequencies. The reason is that the velocity of the high frequency sound is rapid in transmission. The velocity increased in proportion to high frequencies flexes the ossicular chain and consequently reduces the transmission [6].

Transmission of sound is the least disturbed by mass and stiffness, hence, the maximum transfer function of the middle ear is expected to appear in the mid-frequency range such as $1-2 \mathrm{kHz}$. In the experiment, by disconnecting the cochlea with disarticulating the joint between the incus and the stapes, Moller [7] showed that the input impedance at the tympanic membrane fell substantially and found the minimum disturbance in transmission of mid-frequency sound around 1-2 $\mathrm{kHz}$. Therefore, in this frequency region, the transmission will be least affected by factors other than the input impedance of the cochlea itself and consequently keeping the greatest pressure gain of the transfer function of the middle ear.

\section{Changes in middle ear impedance}

There are several examples that cause unique phenomena and hearing loss by changing in middle ear impedance, in relation to mass and stiffness. First, an acoustic reflex that happens by the contraction of the middle ear muscle represents the stiffness-controlled phenomenon, showing more reliable responses below $2 \mathrm{kHz}$. The stiffness limits the transmission of the sounds of low frequency. When it is augmented by a stiffening of the ossicular chain following contraction of the muscle, the low frequency responses are reduced sill further. In contrast, the responses are much less affected at high frequencies, above $1-2 \mathrm{kHz}$, where transmission is not stiffness-controlled [5].

Secondly, otitis media is associated with abnormal increase in mass and stiffness of the middle ear and its progress may physically interrupt the transmission of sound. Early onset of otitis media begins with reduced pressure in the middle ear cavity and increased stiffness of the middle ear structure. The decrease in pressure is commonly a result of dysfunction of the Eustachian tube. Due to dysfunction of the Eustachian tube, reduced pressure appears and stiffens the middle ear structure. As low frequency sound transmission is further disturbed, this early onset of otitis media is expected to cause low-frequency hearing loss and changed tympanogram from a type A to a type C. As otitis media progresses, otorrhea or runny ears may include abnormal increase in mass causing high frequency trans- mission disturbance. Other than low-frequency hearing loss, this stage of otitis media involves high-frequency hearing loss, thus, worse air-conduction hearing thresholds appeared not only at low but also at high frequencies and finally result in type B tympanogram.

Thirdly, otosclerosis is a disease of the bone of otic capsule, specifically at the footplate of the stapes. The stapes becomes "fixed" in the oval window as the disease progresses. Fixation causes stiffness enhancement of the ossicular chain. The audiogram with worse air-conduction thresholds at low frequencies, below $1-2 \mathrm{kHz}$, is typical for otosclerosis [8]. Sometimes, in an audiogram with a drop in bone-conduction (BC) thresholds at $2 \mathrm{kHz}$, a "notch" is noted describing a decrease in cochlear sensitivity. This effect, known as the "Carhart's notch," actually represents the loss of inertial $\mathrm{BC}$ component as a result of fixation [9-11]. Inertial BC, a middle ear involvement of the entire $\mathrm{BC}$ mechanism, will be further discussed to understand this peculiar phenomenon. Although some researchers controversially argued whether there is a recovery in BC thresholds after surgery, the recovery is rather considered the effect of stiffness when the stapes footplate is fixed in position [12].

$\mathrm{BC}$ mechanisms using a bone vibrator basically stimulates hair cells in the cochlear through the bones of the skull. Three primary components in $\mathrm{BC}$ hearing are involved [13]. First component is the $\mathrm{BC}$ through the inner ear. As the BC transducer vibrates the cranial bones, the sound bypasses the eardrum and reaches the cochlear filled with lymphatic fluids. The vibration of temporal bones, the difference of the area between the scala vestibuli and the scala tympani and the capacity of endolymph, induces distortional movement and traveling wave activity of the basilar membrane. This is called distortional BC. Second component is the $\mathrm{BC}$ through the middle ear. When the skull is set into motion, the ossicles move out of phase and cause the same cyclic motion. With the help of inertial force, the stapes move in and out of oval window in the same way as when the sound energy hits the tympanic membrane. This component is called inertial BC. This contribution is only the case when the vibratory energy comes from the mastoid, along the axis of the movement. However, when the vibrator is placed on the forehead, this component is not significant due to the perpendicular axis of the energy access of the vibrator (Fig. 2). Final component is the BC through the external auditory meatus of the outer ear. When a bone vibrator is placed on the skull, the vibration is transmitted to the bone and cartilage portions of the external auditory meatus, creating a sound. Usually, this sound energy escapes from the unoccluded ear through the cartilage portion of the external auditory meatus. However, some of the energy trapped in the bony structure of the meatus hits the tympanic membrane and is combined with the energy 


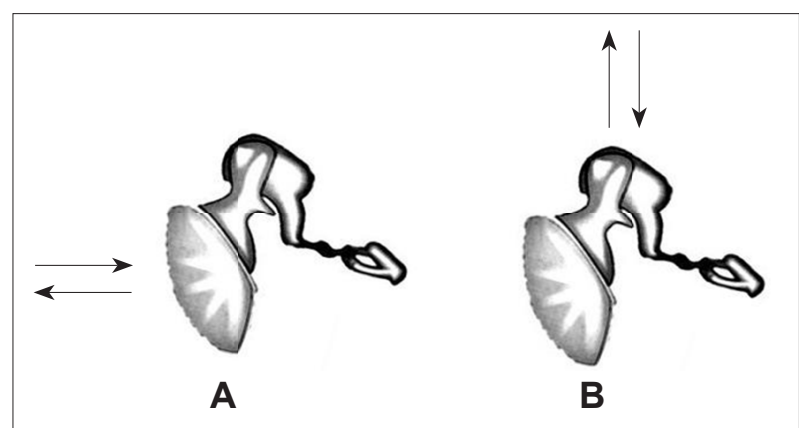

Fig. 2. Vibratory directions of the ossicles according to a bone conduction vibrator placed on the mastoid $(A)$ and on the forehead $(B)$.

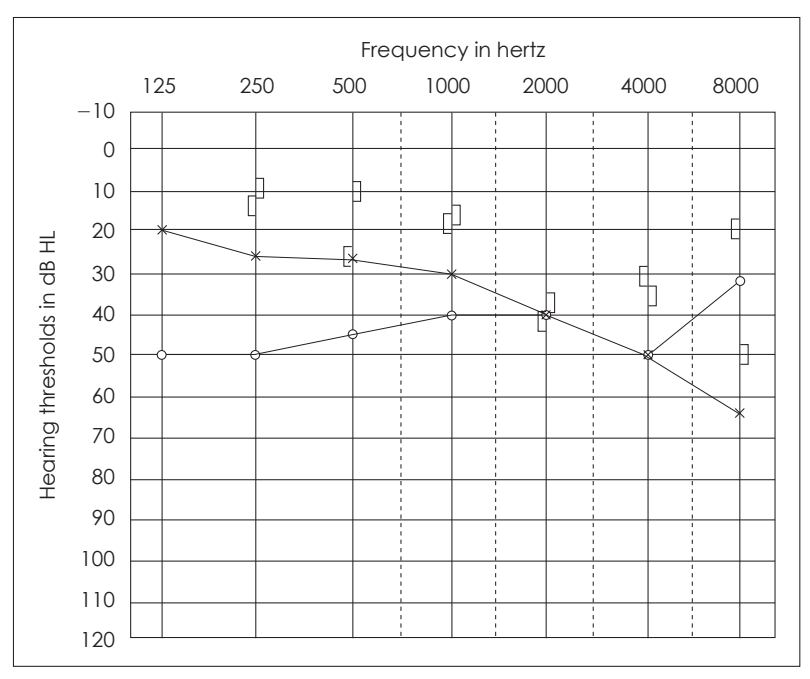

Fig. 3. An example of audiogram in a patient with otosclerosis on the right ear showing Carhart's notch.

from the inertial $\mathrm{BC}$ component and stimulates the inner ear. This component is called osseotympanic BC [8]. Therefore, the vibration of the cranial bones receives not only the response of the inner ear but also air-conduction pathway through the middle ear and the outer ear. Without loss of the inner ear, the middle ear disorder may result in worse $\mathrm{BC}$ hearing thresholds which have appeared in otosclerosis (Fig. 3).

\section{Mass and Stiffness of the Cochlea Partition}

The place and timing principles for the pitch perception

The pitch perception can be explained in the cochlea by two principles, namely the position on the traveling wave in the cochlear partition (place principle) and the temporal pattern of vibrating at anyone point on the wave (timing principle). These two types of principles are based on the selective activity of auditory nerve fibers according to their place of innervations along the cochlear partition and time-pattern of their firing, so called phase-locking, at low frequencies [14]. This means that the spike discharges of auditory nerve fibers are locked to one phase of the stimulation waveform, for example, perhaps once every 100 cycles on average. Apparently, they fire in only one phase of the stimulus sinusoids when they fire. Phase-locking diminishes for frequencies above $2 \mathrm{kHz}$, although $5 \mathrm{kHz}$ is often referred as upper frequency limit and some phase-locked responses are detectable up to $12 \mathrm{kHz}$ with suitable averaging technique [15]. Nevertheless, phase-locking is a sensitive indicator of the activation of an auditory nerve fiber below $5 \mathrm{kHz}$, suggesting that the timing principle might be used in this range of the frequencies. In contrast, place principle is effective above $5 \mathrm{kHz}$ with the notion that a peak of activity of the basilar membrane would be sufficient to evoke a sensation of pitch [5].

\section{Cochlear mechanics of the cochlear partition: a traveling wave}

The movement of the cochlear fluids and the cochlear partition caused by the vibrations of the middle ear bones and oval window displaces the fluid to the round window. This initiates a wave of displacement on the basilar membrane, which then travels from the base to apex. Along with tectorial membrane and organ of Corti, the basilar membrane is a part of the cochlear partition generating unique cochlear movement with its distinctive figure. The basilar membrane of the cochlear partition undergoes an important gradation in dimensions tapering up the opposite figure to the cochlear duct which is broad near the base and narrow towards the apex. Also, the traveling pattern and position of the wave on the basilar membrane operates differently depending on the frequency of the stimulus. The physical mechanics of the basilar membrane and the cochlear fluids determine the decoding frequency. At the base of the cochlea, high frequencies stimuli are decoded with the maximum wave amplitude generated, while low frequencies stimuli are decoded with the maximum wave amplitude generated at the apex of the cochlea (Fig. 4) [16]. This movement of the cochlear partition in human and animal cadavers was examined and introduced as a traveling wave by von Békésy [17]. Thus, different locations are tuned to different stimulus, establishing tonotopicity in the basilar membrane. The tonotopicity maintains the auditory cortex in the temporal lobe. With the notion of characteristic frequency of the auditory nerves, there are the fundamental conceptions of the place principle for pitch perception, which is primarily affected by the gradients of mass and stiffness along the basilar membrane.

\section{Mass and stiffness impact on cochlear mechanics}

Although the cochlear partition maintains the same basic structure along the length of the cochlear, it changes in property as it progresses from the base to apex in three ways. Firstly, the cochlear partition is approximately ten times wider at the base 
Fig. 4. The traveling wave pattern of the basilar membrane for the high $(A)$ and low $(B)$ frequencies and the frequencies producing maximum amplitudes along the basilar membrane. The cochlea at which the traveling wave reaches its maximum amplitude varies with the frequency of the stimulus. At the base of the cochlea, high frequencies stimuli are decoded with the maximum wave amplitude generated, while low frequencies stimuli are decoded with the maximum wave amplitude generated at the apex of the cochlea, sequentially (C).

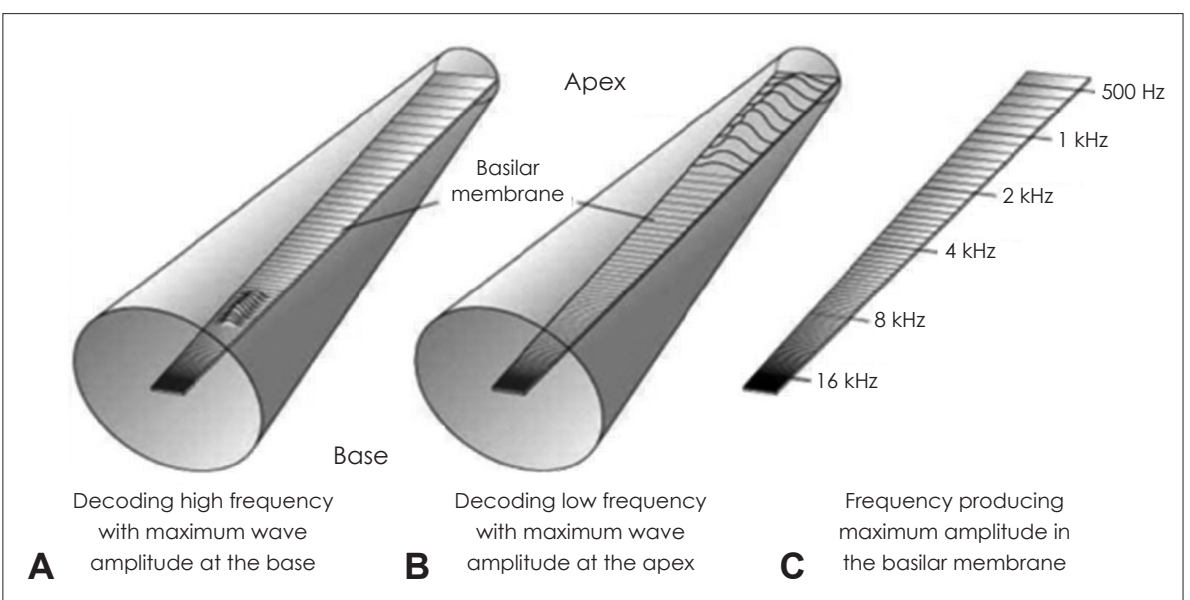

than the apex. Secondly, it has more mass at the base than the apex largely because of increased number and size of supporting cells in the organ of Corti. Thirdly, the cochlear partition is stiffer about one hundredfold at the base than the apex due to the structural characteristics of the basilar membrane as mentioned above. These changes in width, mass, and especially stiffness from the base to apex have the cochlear partition's ability to absorb high frequency energy changes in fluid pressure decline toward the apex. Therefore, different locations are tuned to different stimulus frequencies as mentioned in the traveling wave theory. Moreover, the traveling wave does not maintain a uniform morphology throughout the cochlea due to the structural characteristics of the partition. It increases in amplitude while progressing from the base to apex until it reaches a maximum, beyond which it declines rapidly. The place along the cochlea at which the traveling wave reaches its maximum amplitude varies with the frequency of the stimulus.

The cochlear is a complicated hydro-mechanical system activated by the inward and outward motions of the stapes foot plate; inward motion generates pressure increase of the perilymph near the oval window while outward motion produces decreased pressure. Because the bony cochlear capsule is inflexible and the partition is flexible, the pressure must be absorbed and transmitted across the partition for pressure compensation following bulging of lymphatic fluids. Therefore, the pressure change to the fluid for compensation can be displaced toward both base and apex directions. However, in fact, the direction of the traveling wave appeared in the fashion of baseto-apex always. This was proved with an identical pattern of the traveling wave direction apically even if the stimulation was provided artificially near to the apex. Further, the nature of the traveling wave was not affected by an incision made entirely through the basilar membrane $[17,18]$, proving that a sound energy in the basilar membrane is not transmitted from one region to the adjacent region. Rather, the progression of the transmission occurs based on the whole structural and physical characteristics of the cochlear partition.

\section{Conclusion}

Mass and stiffness of the middle ear and the cochlear partition affect on the peculiar characteristics of transmission of the middle ear and the distinctive behavior of the cochlear mechanics. Mass helps low-frequency vibration and disturbs transmitting high-frequency sound while stiffness helps highfrequency vibration and disturbs transmitting low-frequency sound. Applying this principle, the band-pass characteristic transfer function of the middle ear can be explained. The maximum transfer function of the middle ear, approximately 24-29 $\mathrm{dB}$, at $1-2 \mathrm{kHz}$, are not completely applied due to stiffness of the middle ear structure at lower frequencies and mass of the middle ear bones at higher frequencies. Several examples, an acoustic reflex showing more reliable responses below $2 \mathrm{kHz}$ representing the stiffness-controlled phenomenon, otitis media associating with abnormal increase in stiffness and mass of the middle ear and its progress of the low and high frequency hearing loss involvement, and otosclerosis with fixation of the oval window and stiffness enhancement resulting worse air-conduction thresholds at lower frequencies are discussed. Additionally, the "Carhart's notch" was identified with inertial BC component which is a middle ear involvement of the entire BC mechanisms.

Because the basilar membrane shapes the opposite to the cochlear duct which is broad near the base and narrow towards the apex, the traveling pattern and position of the wave on the basilar membrane operates differently depending on the frequency of the stimulus. At the base of the cochlea, high frequencies stimuli are decoded while low frequencies stimuli are decoded at the apex of the cochlea as introduced in the traveling wave theory by von Békésy [17]. This phenomenon is also 
affected by the structural and physical characteristics of the whole cochlear partition. The cochlear partition is approximately ten times wider at the base than the apex; it has more mass at the base than the apex; it is stiffer about one hundredfold at the base than the apex. These changes in width, mass, and especially stiffness from the base to apex have the cochlear partition's ability to absorb high frequency energy changes in fluid pressure decline toward the apex. Therefore, different locations are tuned to different stimulus frequencies. Due to the whole structural and physical characteristics of the cochlear partition, the direction of the traveling wave was also proved to be in the fashion of base-to-apex always.

\section{REFERENCES}

1) Nedzelnitsky V. Sound pressures in the basal turn of the cat cochlea. J Acoust Soc Am 1980;68:1676-89.

2) Voss SE, Shera CA. Simultaneous measurement of middle-ear input impedance and forward/reverse transmission in cat. J Acoust Soc Am 2004;116(4 Pt 1):2187-98.

3) Aibara R, Welsh JT, Puria S, Goode RL. Human middle-ear sound transfer function and cochlear input impedance. Hear Res 2001;152: $100-9$.

4) O'Connor KN, Puria S. Middle ear cavity and ear canal pressuredriven stapes velocity responses in human cadaveric temporal bones. J Acoust Soc Am 2006;120:1517-28.

5) Pickles JO. An introduction to the physiology of hearing. 4th ed. Bingley, UK: Brill Academic Publishers;2012.

6) Guinan JJ Jr, Peake WT. Middle-ear characteristics of anesthetized cats. J Acoust Soc Am 1967;41:1237-61.
7) Moller AR. An experimental study of the acoustic impedance of the middle ear and its transmission properties. Acta Otolaryngol 1965;60:129-49.

8) Harrell RW. Pure tone evaluation. In: Katz J, Medwetsky L, Burkard RF, Hood LJ, editors. Handbook of Clinical Audiology. 7th ed. Baltimore, USA: Lippincott Williams \& Wilkins;2014. p.71-88.

9) Carhart R. Clinical application of bone conduction audiometry. Arch Otolaryngol 1950;51:798-808.

10) Carhart R. Atypical audiometric configurations associated with otosclerosis. Trans Am Otol Soc 1962;50:153-71.

11) Emanuel DC. Why Does the Carhart Notch appear at $2000 \mathrm{~Hz}$ ? [cited 2015 January 10]. Available from: URL: http://www.audiologyonline.com/ask-the-experts/why-does-carhart-notch-appear-509.

12) Kashio A, Ito K, Kakigi A, Karino S, Iwasaki S, Sakamoto T, et al. Carhart notch 2-kHz bone conduction threshold dip: a nondefinitive predictor of stapes fixation in conductive hearing loss with normal tympanic membrane. Arch Otolaryngol Head Neck Surg 2011;137:236-40.

13) Tonndorf J. A new concept of bone conduction. Arch Otolaryngol 1968;87:595-600.

14) Moore B. An Introduction to the Psychology of Hearing. 5th ed. London, UK: Academic Press;2003.

15) Recio-Spinoso A, Temchin AN, van Dijk P, Fan YH, Ruggero MA. Wiener-kernel analysis of responses to noise of chinchilla auditorynerve fibers. J Neurophysiol 2005;93:3615-34.

16) von Békésy G. On the resonance curve and the decay period at various points on the cochlear partition. J Acoust Soc Am 1949;21: 245-54.

17) von Békésy G. Experiments in hearing. New York, USA: McGrawHill;1960.

18) Ryan AF. New Views of Cochlear Function. In: Robinette MS, Glattke TJ, editors. Otoacoustic Emissions: Clinical Applications. New York, USA: Thieme;1997. p.22-45. 